$01,05,16$

\title{
Влияние отжига в ферромагнитном состоянии на структуру сплава железа с 18 at.\% галлия
}

\author{
(C) Ю.П. Черненков ${ }^{1}$, Н.В. Ершов ${ }^{2, \uparrow}$, В.А. Лукшина ${ }^{2,3}$ \\ ${ }^{1}$ Петербургский институт ядерной физики, НИЦ „Курчатовский институт“, \\ Гатчина, Россия \\ ${ }^{2}$ Институт ффизики металлов УрО РАН, \\ Екатеринбург, Россия \\ ${ }^{3}$ Уральский федеральный университет им. Б.Н. Ельцина, \\ Екатеринбург, Россия \\ ฯ E-mail: nershov@imp.uran.ru
}

(Поступила в Редакцию 26 июня 2018 г.)

\begin{abstract}
Атомная структура образцов сплава железо-галлий, содержащего около 18 at.\% галлия, исследовалась методом рентгеновской дифракции. Образцы отжигались в парамагнитном $\left(T>T_{\mathrm{C}}\right)$ и в ферромагнитном $\left(T<T_{\mathrm{C}}\right)$ состояниях. В первом случае структурное состояние фиксировалось закалкой от температуры отжига в воду, во втором - медленным охлаждением. Структурные исследования монокристаллических образцов проводились на четырехкружном рентгеновском дифрактометре при комнатной температуре. Из данных рентгеновской дифракции следует, что независимо от способа термообработки сплава в нем присутствуют B2-кластеры, локально упорядоченные области со структурой типа $\mathrm{CsCl}$, ранее обнаруженные нами в сплавах железа с кремнием (до 10 at.\% $\mathrm{Si}$ ) и с алюминием (7at.\% Al). Кроме B2-кластеров, в закаленном образце наблюдаются области с $D 0_{3}$-ближним порядком, размер которых после отжига в ферромагнитном состоянии значительно увеличивается, то есть возникает дальний порядок. Обсуждается связь тонких структурных изменений сплава при различных термообработках с его магнитоупругими и магнитострикционными свойствами.
\end{abstract}

Работа выполнена в рамках государственного задания по теме „Магнит“ № АААА-А18-118020290129-5 и проекту № 18-10-2-5 программы УрО РАН при поддержке РФФИ (грант № 18-02-00391).

DOI: 10.21883/FTT.2019.01.46889.174

\section{1. Введение}

Богатые железом сплавы железа и галлия стали объектами, привлекающими значительный научный интерес после того, как на конференции Intermag 2000 (Toronto, Canada) A.E. Clark и др. впервые сообщили об обнаружении более чем десятикратного увеличения магнитострикции за счет добавления галлия в железо, максимальная величина которой достигается при концентрации галлия около 17 at.\% [1]. Позднее, в 2003 г., они привели результаты измерений коэффициента тетрагональной магнитострикции $\lambda_{100}$ в монокристаллических образцах сплава железо-галлий в зависимости от концентрации (4-35 at.\% Ga) и условий их термообработки [2]. Как показано на рис. 1, заимствованном из этой публикации, примерно до 17 at.\% Ga коэффициент $\lambda_{100}$ увеличивается пропорционально квадрату концентрации галлия и не зависит от того, был ли образец медленно охлажден или закален в воду после выдержки при температуре $1000^{\circ} \mathrm{C}$. Между 17 и 19 at.\% Ga величина магнитострикции продолжает расти в закаленных образцах и начинает убывать в медленно охлажденных образцах. При максимальном значении $3 / 2 \lambda_{100}$ в $400 \times 10^{-6}$ разность достигает $\sim 25 \%$.

В соответствии с фазовой диаграммой сплавов железо-галлий [3] при концентрациях галлия около 18 at.\% и температурах, превышающих $750^{\circ} \mathrm{C}$, сплавы находятся в парамагнитном состоянии и в области A2-фазы или неупорядоченного твердого раствора галлия в $\alpha$-железе. При понижении температуры они переходят в ферро-

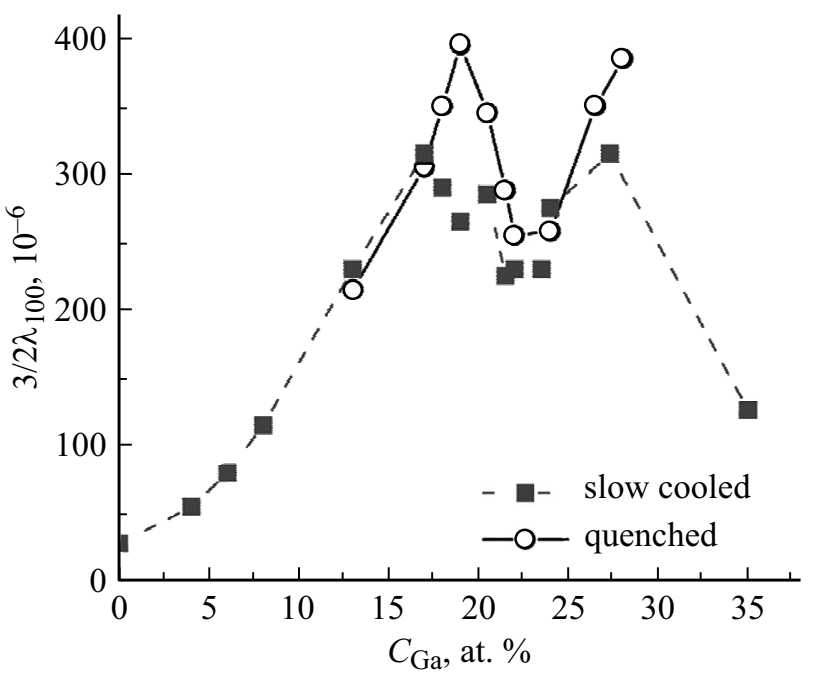

Рис. 1. Зависимость коэффициента тетрагональной магнитострикции $\lambda_{100}$ сплава железо-галлий, измеренная в магнитном поле $H=15 \mathrm{kOe}$, от концентрации галлия $C_{\mathrm{Ga}}$ и условий термообработки [2]. 

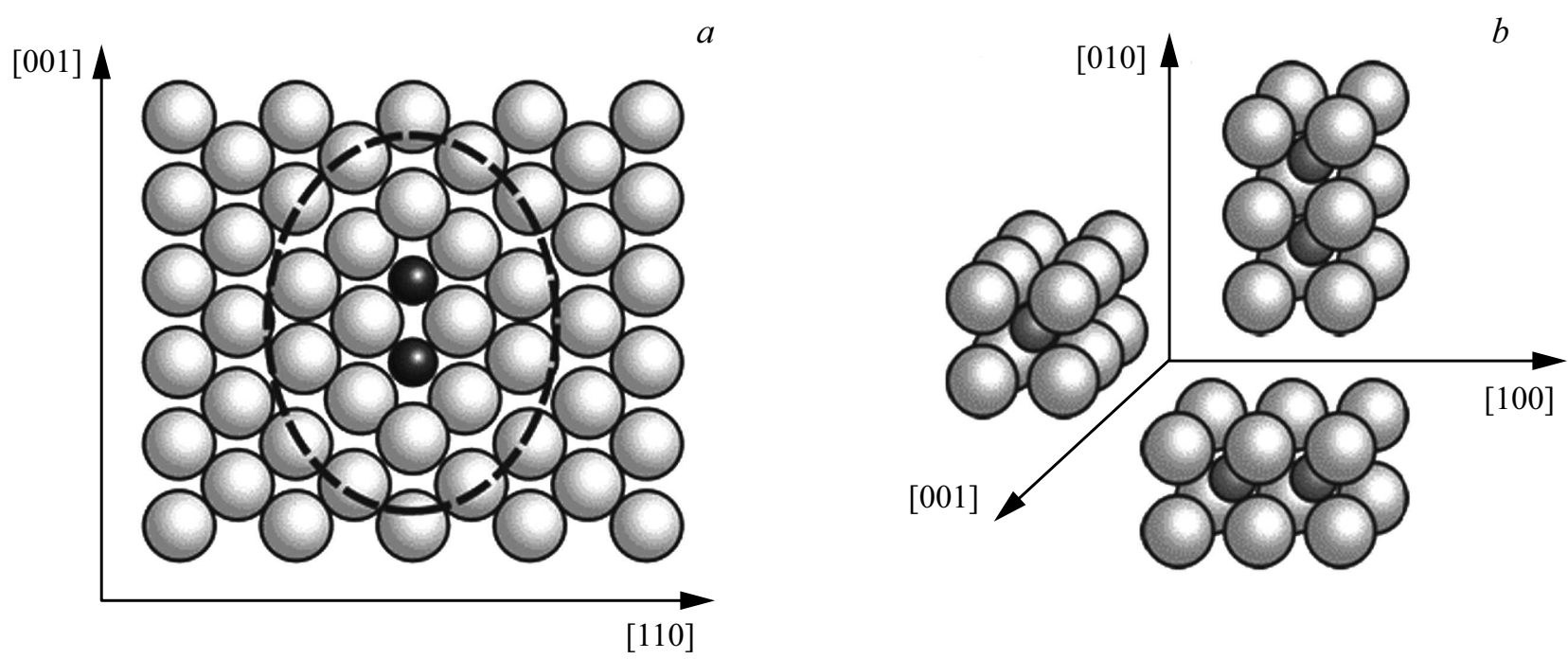

Рис. 2. Схематическое изображение В2-кластера в сечении плоскостью (110) (a) и возможные ориентации $B 2$-кластеров вдоль осей $\langle 100\rangle(b)$.

магнитное состояние и двухфазную область, где наряду с A2-фазой сосуществует вторая - $\mathrm{Fe}_{3} \mathrm{Ga}$ со структурой типа $\mathrm{DO}_{3}$.

Первопринципные исследования химического упорядочения в $\mathrm{Fe}-\mathrm{Ga}-$-плавах показали [4], что при переходе сплава из парамагнитного в ферромагнитное состояние эффективная энергия взаимодействия $\mathrm{Ga}-\mathrm{Ga}$ на расстоянии первых и третьих соседей уменьшается, а на расстоянии вторых - увеличивается. Этот результат предсказывает образование структуры типа $B 2$ в парамагнитном состоянии. Моделирование структуры сплава при 18 at.\% галлия методом Монте-Карло в рамках тех же исследований показало, что в ферромагнитном состоянии должен формироваться ближний порядок $D 0_{3}$, где атомы галлия являются третьими соседями.

Поскольку магнитострикция является структурно обусловленным эффектом, то важную роль в формировании гигантской магнитострикции в сплаве железо-галлий должны играть особенности упорядочения атомов галлия и изменения структуры в зависимости от термической предыстории. С помощью первопринципных расчетов было показано [5], что В2-структура является гораздо более перспективной для увеличения коэффициента магнитострикции при увеличении содержания галлия по сравнению с $D 0_{3}$. Авторы работы [6] использовали модель случайно ориентированных пар атомов галлия, являющихся вторыми соседями и представляющими элемент ближнего B2-порядка, для объяснения эффекта уменьшения магнитной анизотропии в сплаве железо-галлий. По их мнению, это является результатом конкуренции между кристаллической анизотропией и анизотропией флуктуаций намагниченности, обусловленной локальной анизотропией $B 2$-пар $\mathrm{Ga}-\mathrm{Ga}$. B настоящее время природа гигантской магнитострикции еще является предметом дискуссии, однако в [6] делается предположение, что существование таких пар $\mathrm{Ga}-\mathrm{Ga}$ и является причиной увеличения магнитострикции, поскольку ее величина изменяется пропорционально квадрату концентрации галлия в $\alpha$-FeGa.

Атомистическое моделирование формирования наноструктур в сплаве $\mathrm{Fe}-\mathrm{Ga}$ при 19 at.\% Ga показало [7], что неупорядоченный твердый раствор галлия (фаза $A 2$ ) может распадаться по схеме $A 2 \rightarrow B 2 \rightarrow D 0_{3}$ c образованием на промежуточном этапе нанообластей $B 2$-типа размером от 3 до $10 \mathrm{~nm}$. Однако по данным рентгеновской дифракции высокого разрешения для сплава с 19 at.\% Ga [8] в закаленных образцах ближний порядок не наблюдается, а в медленно охлажденных - области c $\mathrm{DO}_{3}$-порядком занимают примерно треть объема и сосуществуют с неупорядоченной фазой $A 2$. Исследования структуры медленно охлажденных монокристаллов сплавов $\mathrm{Fe}_{81.6} \mathrm{Ga}_{18.4}$ и $\mathrm{Fe}_{81} \mathrm{Ge}_{9}$ методом рентгеновской дифракции показали [9], что ближний порядок в расположении атомов $\mathrm{Ga}$ соответствует $D 0_{3}$-типу, а атомы $\mathrm{Ge}$ предпочитают химическое упорядочение B2-типа. Поэтому авторы предположили, что именно $D 0_{3}$-локальное упорядочение важно для усиления магнитоупругости в сплавах на основе железа.

Исследования локального упорядочения в сплавах железо-кремний показали [10-12], что в закаленных образцах сплава, содержащего 5-10 at.\% $\mathrm{Si}$, имеются кластеры с B2-упорядочением. При увеличении концентрации кремния от 5-6 до 8 at.\% появляются небольшие области с $D 0_{3}$-порядком, размеры и объемная доля которых растет после 10 минутной выдержки при температуре $450^{\circ} \mathrm{C}$. При 10 at.\% кремния $D 0_{3}$-фаза представляет собой области с дальним упорядочением [12]. $B 2$-кластеры схематически показаны на рис. 2 и их можно представить, как „ядро“, состоящее из двух $B 2$-ячеек, имеющих общую грань, окруженное растянутыми ячейками $\alpha$-железа. B2-кластеры имеют анизотропную форму - они более протяженные вдоль одной из осей 
легкого намагничивания $\langle 100\rangle$. Области с аналогичным локальным упорядочением обнаружены в монокристаллах сплава железо-алюминий с 7 at.\% $\mathrm{Al}$ [13].

Эти результаты были получены из данных рентгеновской дифракции, то есть после анализа углового распределения интенсивности слабого, но очень информативного диффузного рассеяния. При этом для выделения вклада в рассеяние, обусловленного тепловым движением атомов, проводились измерения при низких температурах [14]. Компьютерное моделирование диффузного рассеяния подтвердило выводы, полученные из анализа экспериментальных данных. Модельные расчеты позволили так же определить количественные соотношения в атомной структуре ближнего порядка - количество и тип атомов, их смещения из узлов идеальной решетки, которые дают хорошее согласие с картиной двумерного распределения интенсивности диффузного рассеяния.

Цель проведенных нами исследований состояла в том, чтобы, используя аналогичный подход, выявить тонкие особенности атомной структуры сплава железо-галлий, подвергнутого разным термообработкам, и связать их с изменением магнитных свойств, магнитоупругости и магнитострикции.

\section{2. Образцы и методика эксперимента}

Рентгеноструктурные исследования проводились на монокристаллических образцах сплава $\mathrm{Fe}-18$ at.\% $\mathrm{Ga}$. Образцы сплава были вырезаны из монолитного кристалла, выращенного про методу Бриджмена, в виде тонких дисков (толщина $0.3 \mathrm{~mm}$, диаметр $8-9 \mathrm{~mm}$ ) с „госсовской“ ориентацией кристаллографических осей, когда оси [110] и [001] лежат в плоскости диска. Образцы сплава были подвергнуты рафинирующему вакуумному отжигу при температуре $1050^{\circ} \mathrm{C}$ в течение $4 \mathrm{~h}$. Первый образец после десятиминутной выдержки в парамагнитном состоянии при температуре $T_{\text {an }}=850^{\circ} \mathrm{C}$ был закален в воду комнатной температуры (скорость закалки не менее $400^{\circ} \mathrm{C} / \mathrm{s}$ ). Предполагается, что высокотемпературная выдержка должна приводить к разупорядочению атомов галлия в решетке железа, а закалка фиксировать разупорядоченное состояние. Второй образец был медленно охлажден после отжига в течение $1 \mathrm{~h}$ в ферромагнитном состоянии. Температура отжига $\left(T_{\text {an }}=450^{\circ} \mathrm{C}\right)$ существенно ниже точки Кюри сплава $\left(T_{\mathrm{C}} \approx 700^{\circ} \mathrm{C}\right)$, но достаточна для активации высокой диффузии галлия в железе для быстрого достижения равновесного состояния.

Рентгеновские дифрактограммы образцов сплава, измеренные при сканировании вдоль различных кристаллографических направлений, сравнивали с аналогичными сканами для монокристалла железа [13]. Это позволило надежно выделить вклад, обусловленный рассеянием на областях с локальным упорядочением примесных атомов $\mathrm{Ga}$, а также исключить вклад рассеяния от характерных для ОЦК-решетки диффузных плоскостей и стержней, обусловленных тепловым движением атомов [14].

Измерения проводились при комнатной температуре на четырехкружном лабораторном дифрактометре (ПИЯФ НИЦ „Курчатовский институт“) в геометрии на просвет. Характеристическое излучение рентгеновской трубки с Мо-анодом $(\lambda=0.071 \mathrm{~nm})$ монохроматизировалось с помощью кристалла пиролитического графита. Рассеянное излучение регистрировалось энергодисперсионным $\mathrm{Si}(\mathrm{Li})$-детектором [10], что позволяет значительно улучшить соотношение сигнал/фон.

\section{3. Результаты и обсуждение}

ОЦК-решетка сплава $\alpha$-FeGa также, как решетка чистого $\alpha$-железа, проявляются в дифракции набором сильных узких рефлексов с четной суммой индексов $h, k, l$, например, (110), (200), (211), (220), (310), (400) и др. Рефлексы с другими сочетаниями целых индексов являются запрещенными. При $B 2$ - или $D 0_{3}$-упорядочении в дифрактограммах сплава появляются сверхструктурные пики, у которых $h+k+l-$ нечетное число, то есть пики с индексами (100), (300), (111) и др. Кроме того, в результате удвоения ячейки вдоль кристаллографических осей при образовании $D 0_{3}$-фазы появляются сверхструктурные пики (рефлексы) с полуцелыми индексами $h=n / 2 k=m / 2 l=p / 2$, где $n, m, p$ - целые нечетные числа, например, (0.5 0.50 .5$),(1.50 .50 .5)$, (1.5 1.50 .5$),\left(\begin{array}{lll}1.5 & 1.5 & 1.5\end{array}\right)$ и т.п.

При переходе от $\alpha$-железа к сплаву железо-галлий подобно тому, как это происходит в случае сплавов железа с алюминием [13], основные брэгговские рефлексы остаются практически неизменными и лишь смещены в меньшие углы рассеяния из-за увеличения параметра ОЦК-ячейки $a$ с ростом концентрации галлия [15]. Из угловых положений пиков в дифрактограммах можно получить параметр решетки для первого образца $a=0.2901(2) \mathrm{nm}$ и для второго $a=0.2896(2) \mathrm{nm}$ (в $\alpha$-железе $a=0.2866 \mathrm{~nm}$ ). Параметры решетки образцов сплава на $1 \%$ больше, чем в $\alpha$-железе, и в пределах двух-трех стандартных отклонений совпадают.

Дифрактограммы, измеренные при $\theta-2 \theta$-сканировании обратного пространства вдоль осей [0 01$]$, [ [ 1111$]$, [110] и [22 1], приведены на рис. 3 с использованием логарифмической шкалы для интенсивности. Сильные ОЦК-отражения (0 0 2), (2 22), (1 110$)$ и (2 20) примерно на четыре порядка интенсивнее, чем интересующее нас диффузное рассеяние. Примеры последнего показаны на рисунке в промежутках между прямым пучком и брэгговскими пиками (002), (222), (1 1 0) и (4 4 1), и между брэгговскими пиками $(002)$ и (004), (1 1 0$)$ и (220) в соответствующих сканах $a, b, c$ и $d$.

В $\theta-2 \theta$-сканах, измеренных вдоль направлений [1 111$]$ и [221], наблюдаются широкие диффузные пики. Они появляются в тех местах, где траектория скана в обратной решетке кристалла пересекает плоскости теплового 


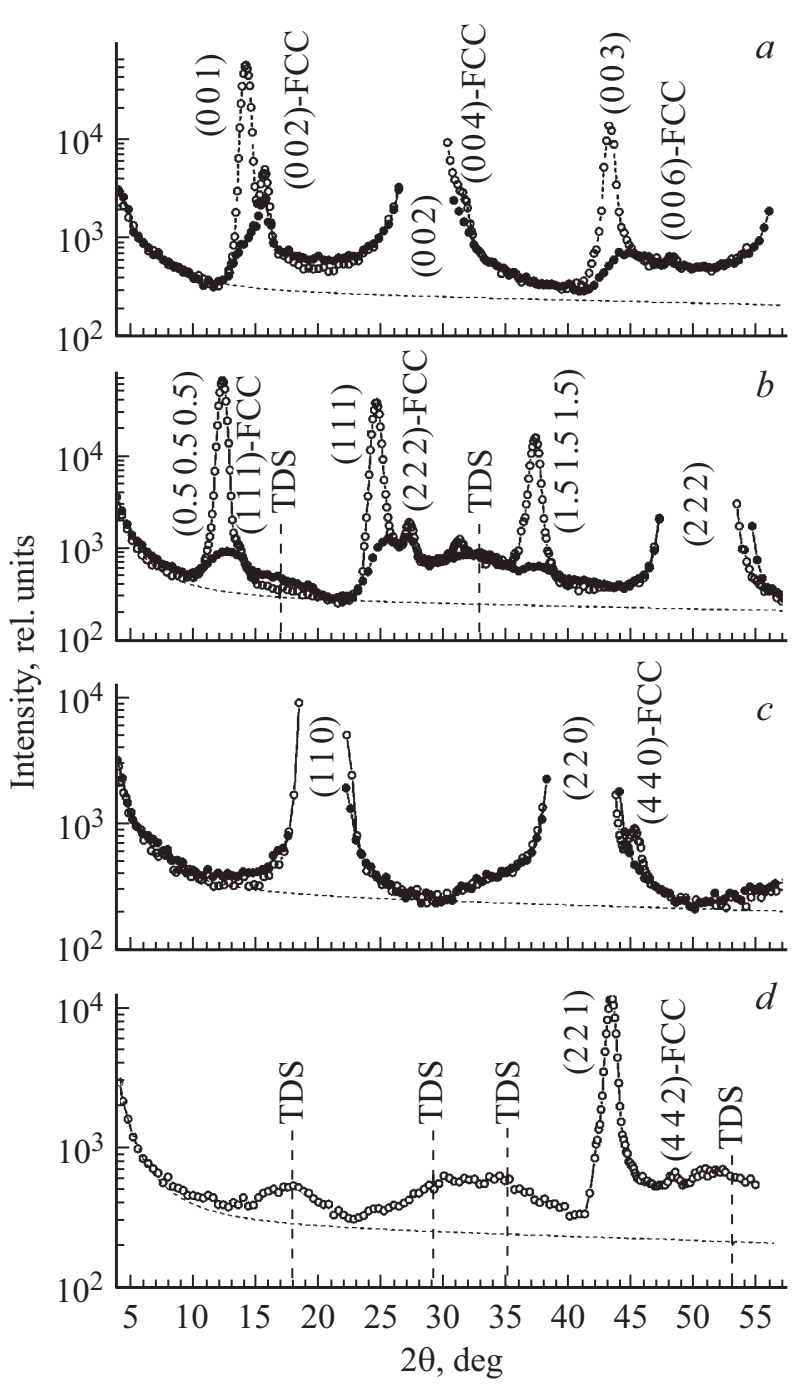

Pис. 3. Дифрактограммы закаленного (сплошные кружки) и отожженного (полые) образцов, измеренные при сканировании вдоль направлений $\left[\begin{array}{lll}0 & 0 & 1\end{array}\right]-a,\left[\begin{array}{lll}1 & 1 & 1\end{array}\right]-b,\left[\begin{array}{ll}1 & 1\end{array}\right]-c$ и $[221]-d$. Величина экспериментальной ошибки примерно равна размеру символа. Фон - пунктирная линия, полученная из МНК аппроксимации экспериментальных точек.

диффузного рассеяния (TDS - отмечены вертикальными штриховыми линиями на рис. 3). TDS-плоскости, характерные для кристаллов с ОЦК-структурой, обусловлены тепловыми колебаниями цепочек атомов [1 11 l] вдоль самих себя [14]. Широкие пики TDS наблюдаются в аналогичных сканах и для монокристалла чистого железа, как показано нами ранее (см. рис. 5, $b$ [13]).

Дифрактограммы образца сплава $\mathrm{Fe}-\mathrm{Ga}$, подвергнутого отжигу в ферромагнитном состоянии, содержат значительно больше деталей, чем дифрактограммы закаленного образца. После отжига появляются узкие сверхструктурные пики (0 01$)$ и (0 03 3), которые наблюдаются на дифрактограмме, измеренной при сканировании вдоль оси [001] (рис. 3,a), одновременно с аналогичными пиками $(0.50 .50 .5),(111)$ и (1.5 1.51 .5$)$ в дифрак- тограмме, измеренной при сканировании вдоль оси [111] (рис. 3,b). Все эти пики происходят от довольно больших областей $D 0_{3}$-фазы. Такое же происхождение имеет узкий пик (221) в дифрактограмме отожженного образца, измеренной при сканировании вдоль оси [22 1] (рис. 3, $d$ ).

В дифрактограммах, приведенных на рис. 3, кроме вкладов от ОЦК- и $D 0_{3}$-фаз наблюдаются узкие рефлексы новой фазы, обозначенные как FCC. Пики от нее слабо выражены в дифрактограммах от закаленного образца, но гораздо более отчетливо проявляются в дифрактограммах отожженного образца, $(h k l)$-FCC. Из погасания и положений более чем десятка таких пиков можно однозначно установить, что элементарная ячейка этой фазы кубическая и ее оси совпадают с осями ОЦК-ячейки, что она гранецентрированная (FCC), а параметр ее ячейки $\sim 0.52 \mathrm{~nm}$.

Для определения вкладов рассеяния от различных фаз, возникающих в сплаве железо-галлий, дифрактограммы описывались совокупностью пиков, форма которых задавалась функциями Гаусса и Лоренца, а их параметры - положение, ширина и высота - определялись методом наименьших квадратов (МНК) при согласовании расчетных и экспериментальных данных. Основной интерес представляет вклад в рассеяние от $B 2$ - и $D 0_{3}$-фаз после закалки и отжига образцов сплава. Очевидно, что этот вклад небольшой по величине в случае закаленного образца, и также небольшой вклад от B2-фазы в случае отожженного образца, поэтому для повышения надежности МНК-процедуры необходимо корректно описать монотонно убывающий фон. Для этого из нескольких $\theta-2 \theta$-сканов, включая [0 01$]$, [1 1 1] и [1 10$]$, для отожженного образца в диапазоне $5^{\circ}<2 \theta<55^{\circ}$ был взят целиком участок $5^{\circ}<2 \theta<8^{\circ}$ и самые низкие экспериментальные точки на бо́льших углах. Через них с помощью МНК была проведена плавная огибающая, представляющая собой сумму наклонной прямой и функции Лоренца с максимумом в $2 \theta=0$. Результат показан штриховой линией на рис. 3 . В дальнейшем при обработке дифрактограмм параметры фона не варьировались.

Узкие сверхструктурные пики (001), (003), (1 111$)$, (0.5 0.50 .5$),(1.51 .51 .5)$ и (221) в дифрактограмме образца, отожженного в ферромагнитном состоянии, обусловлены дальним $D 0_{3}$-упорядочением. Интенсивность даже самых сильных из них на два порядка меньше, чем у ОЦК-пиков. В дифрактограмме закаленного образца некоторые из них, такие как (0 01$),\left(\begin{array}{ll}1 & 1\end{array}\right),\left(\begin{array}{l}0.5 \\ 0.5\end{array} 0.5\right)$ и (1.5 1.5 1.5), проявляются как широкие диффузные пики (рис. 3).

Результат разложения дифрактограмм, измеренных в направлении [001], от закаленного и отожженного образцов показан на рис. 4. Как и при описании фона, склоны сильных ОЦК-пиков (002) и (004), а также (222) в скане вдоль оси [1 11 1], описывались „крылом“ функции Лоренца, параметры которой варьировались. 


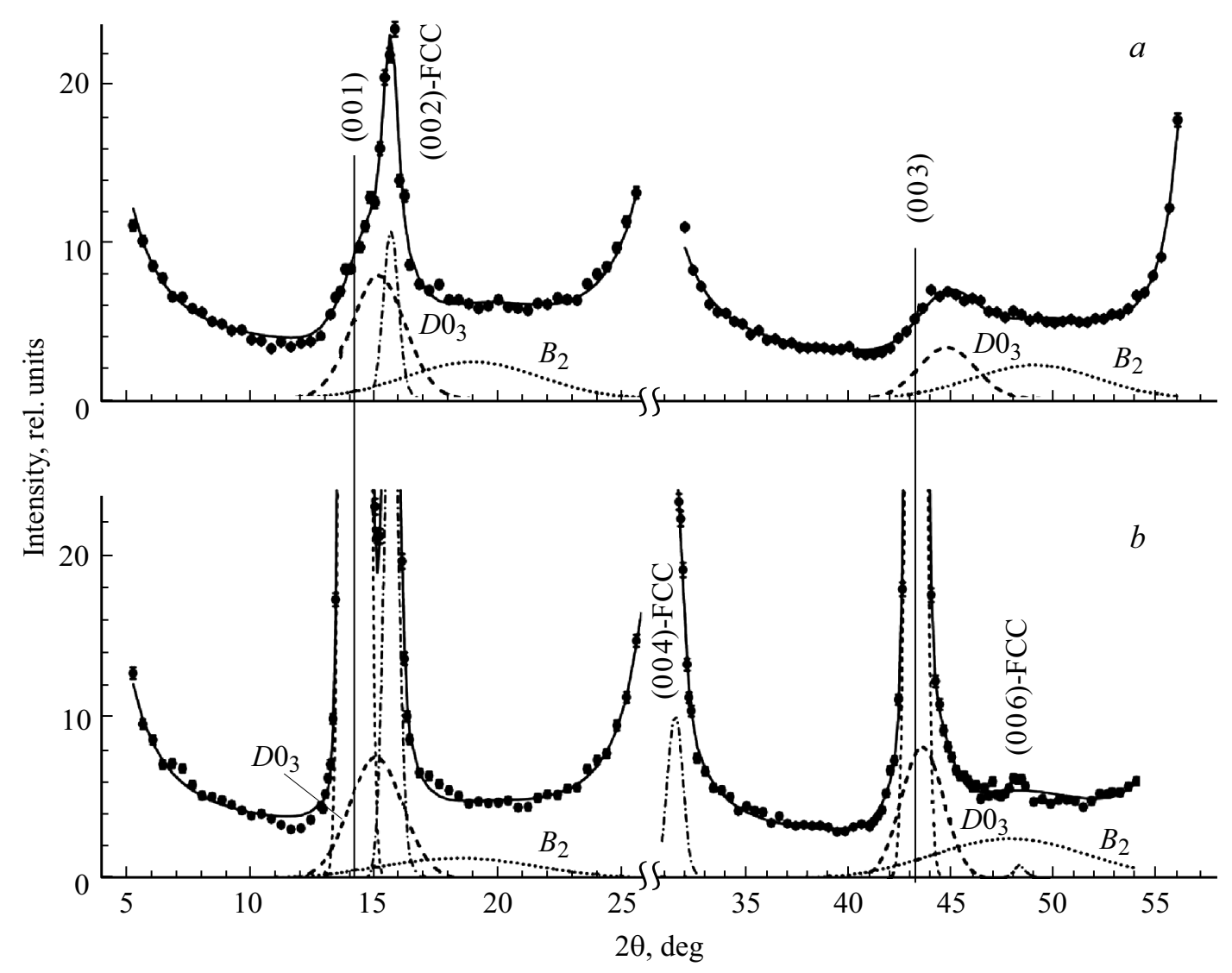

Рис. 4. Угловое распределение интенсивности рассеяния закаленного $(a)$ и отожженного $(b)$ образцов сплава, измеренное при сканировании вдоль оси [001]. Вклад от рассеяния на $B 2$-кластерах, областях с $D 0_{3}$-упорядочением и областях с FCC-фазой в виде отдельных пиков, нанесенных разными линиями.

Для каждой из дифрактограмм на рисунке показаны суммарная кривая, огибающая экспериментальные точки, вклады от $B 2-$ и $D 0_{3}$-фаз и пики $(002),\left(\begin{array}{ll}0 & 0\end{array}\right)$ и (006) FCC-фазы. Полученные разложения не позволяют получить количественные характеристики фаз, но дают их качественную оценку и позволяют выяснить тенденцию их изменения. Если ширина сильных $D 0_{3}$-пиков в дифрактограмме отожженного образца примерно $0.7^{\circ}$, то у диффузных $B 2$ - и $D 0_{3}$-пиков в дифрактограмме закаленного образца она значительно больше, $\sim 7^{\circ}$ и $\sim 3^{\circ}$ соответственно. Узкими являются FCC пики (ширина $\sim 0.9^{\circ}$ ), что говорит о больших размерах областей этой фазы, но, судя по интенсивности, их объемная доля мала. После отжига образца в ферромагнитном состоянии пики от В2-фазы изменяются незначительно, а в расчетном положении для фазы $D 0_{3}$ с дальним порядком возникают узкие и интенсивные пики (001) и (003). Однако их асимметрия, отчетливо проявляющаяся на правом „крыле“ (003) (рис. 4,b), вынуждает оставлять в разложении дифрактограммы диффузные $D 0_{3}$-пики, очень похожие на те, которые выделены в дифрактограмме от закаленного образца (рис. 4,a). По-видимому, после отжига отчасти остаются небольшие $D 0_{3}$-кластеры, присутствие которых однозначно определяется в зака- ленном образце. При этом необходимо отметить важный факт - в отличие от сплавов $\mathrm{Fe}-\mathrm{Al}$ (7 at.\% Al) [13] и

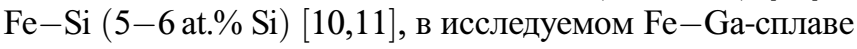
B2-пики смещены не в меньшие, а в большие углы

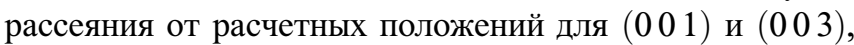
отмеченных вертикальными линиями на рис. 4.

Из ширины пика, используя формулу Шеррера [16], можно получить оценку среднего размера упорядоченной области (кластера). Для кластеров B2-фазы в обоих образцах она $\sim 0.6-0.7 \mathrm{~nm}$, что соответствует примерно двум элементарным ОЦК-ячейкам. Области с $D 0_{3}$-упорядочением в образце сплава, закаленном после отжига в парамагнитном состоянии, имеют размер $\sim 1.6 \mathrm{~nm}$, что составляет длину двух-трех элементарных $D 0_{3}$-ячеек, а в образце, отожженном в ферромагнитном состоянии, их размер увеличивается до $\sim 20 \mathrm{~nm}$. Изменения атомной структуры сплава железо-галлий находятся в соответствии с ранее предсказанными тенденциями в формировании ближнего порядка при отжиге в зависимости от магнитного состояния [4].

Аналогично тому, как это было сделано для дифрактограмм, полученных при сканировании вдоль оси [0 0101$]$ (рис. 4), с помощью МНК проведено разложение дифрактограмм для направления [l 111 l. Результат разложе- 


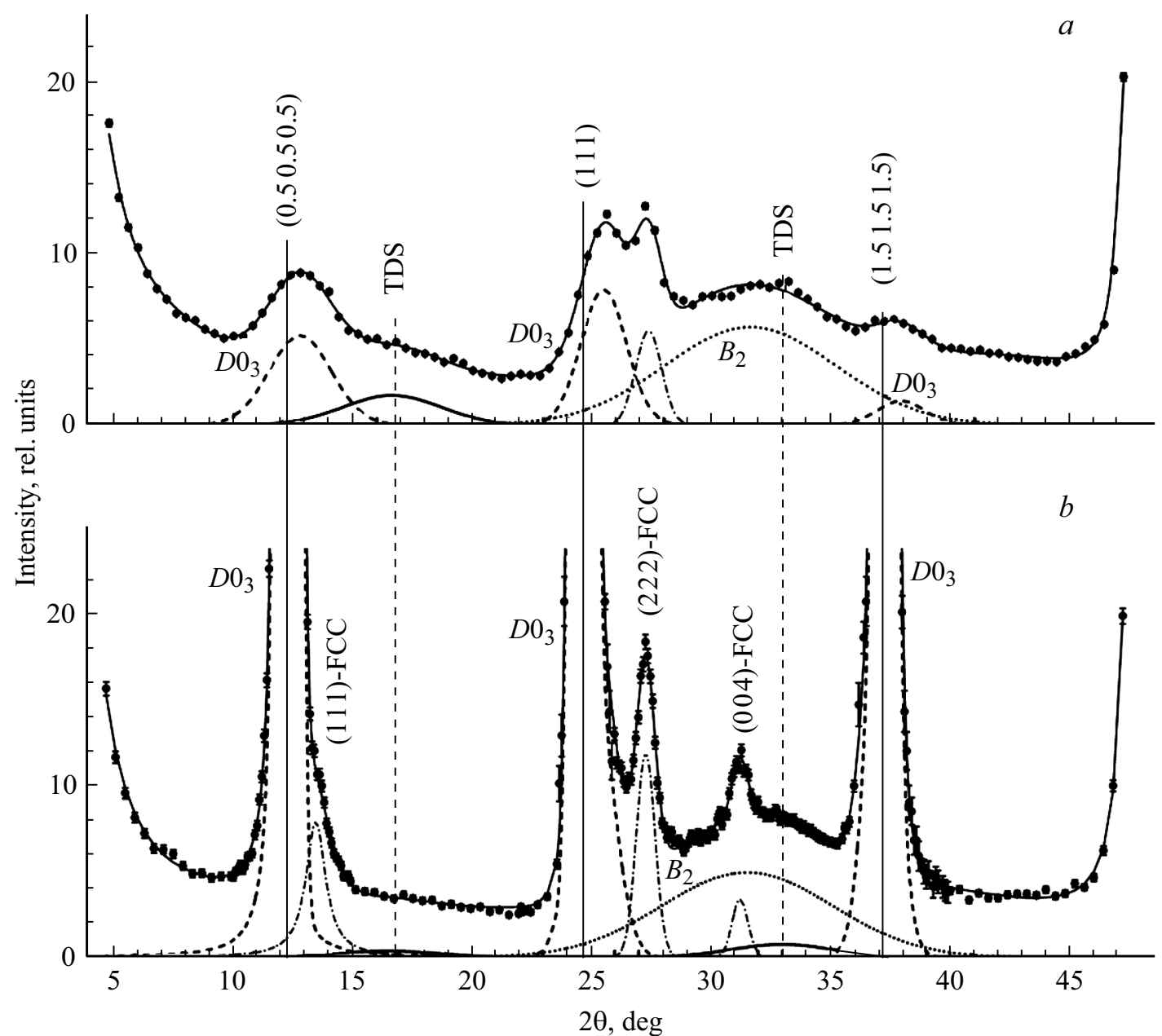

Рис. 5. То же, что на рис. 4, измеренные при сканировании вдоль оси [1 111 . Вклад от рассеяния на В2-кластерах, областях с $D 0_{3}$-упорядочением и областях с FCC-фазой в сплаве показан отдельными пиками.

ния показан на рис. 5. Следует отметить, что в случае закаленного образца все диффузные $D 0_{3}$-пики $\left(\begin{array}{lll}0.5 & 0.5 & 0.5\end{array}\right)$, (1 111$)$ и $(1.51 .51 .5)$, приведенные на рис. 5, $a$, точно также как пики (001) и (003), приведенные на рис. 4, $a$, несколько смещены в сторону больших углов рассеяния из расчетных положений, отмеченных сплошными вертикальными линиями. Следовательно, параметр решетки небольших $D 0_{3}$-кластеров, сформировавшихся в сплаве в парамагнитном состоянии, меньше параметра $D 0_{3}$-ячейки в больших областях $\mathrm{DO}_{3}$-фазы.

Заметное уменьшение интенсивности диффузного рассеяния, показанного на рис. 3, наблюдается после отжига в ферромагнитном состоянии в скане вдоль оси [ $\left.\begin{array}{lll}1 & 1 & 1\end{array}\right]$ на участках $5^{\circ}<2 \theta<10^{\circ}$ и $14^{\circ}<2 \theta<20^{\circ}$, а в скане вдоль оси $\left[\begin{array}{lll}0 & 0 & 1\end{array}\right]$ на участке $17^{\circ}<2 \theta<23^{\circ}$. Если при $\omega$-сканировании, то есть перпендикулярно к направлению $\theta-2 \theta$-скана, $D 0_{3}$-пики в случае отожженного образца узкие, как пик (111) на рис. 6, $a$, то диффузное рассеяние имеет большую протяженность в случае обоих образцов. Например, на рис. $6, b$ показан $\omega$-скан перпендикулярно оси [001] при $2 \theta=20.4^{\circ}$ для закаленного образца, откуда видно, что интенсивность не меняется во всем диапазоне измерений. Из этого наблюдения можно сделать предположение, что уменьшение вклада диффузного рассеяния после отжига связано с перераспределением атомов Ga между A2-фазой, где атомы Ga одиночные, и фазами $D 0_{3}, \mathrm{FCC}$, в которых формируется дальний порядок, и, возможно, с небольшим увеличением количества $B 2$-кластеров.

После отжига имеет место небольшое уменьшение интенсивности диффузного рассеяния в диапазоне $10^{\circ}<2 \theta<18^{\circ}$ при сканировании вдоль оси [1 10$]$, как показано на рис. 3,c. В то же время на угле $2 \theta \sim 45^{\circ}$ возникает пик (440)-FCС. Как и в случае сплавов $\mathrm{Fe}-\mathrm{Si}[10-12]$ и $\mathrm{Fe}-\mathrm{Al}$ [13] при сравнении с аналогичным сканом для монокристалла железа никаких особенностей, кроме некоторой асимметрии фона возле пика (2 20), не наблюдается и в сплаве $\mathrm{Fe}-\mathrm{Ga}$. Вероятнее всего эта асимметрия в виде приподнятости левого „крыла“ пика (220) в районе $2 \theta \sim 35^{\circ}$ обусловлена вкладом диффузного рассеяния.

Прежде чем перейти к обсуждению полученных результатов отметим еще раз одно важное обстоятельство. Присутствие сверхструктурных пиков диффузного 

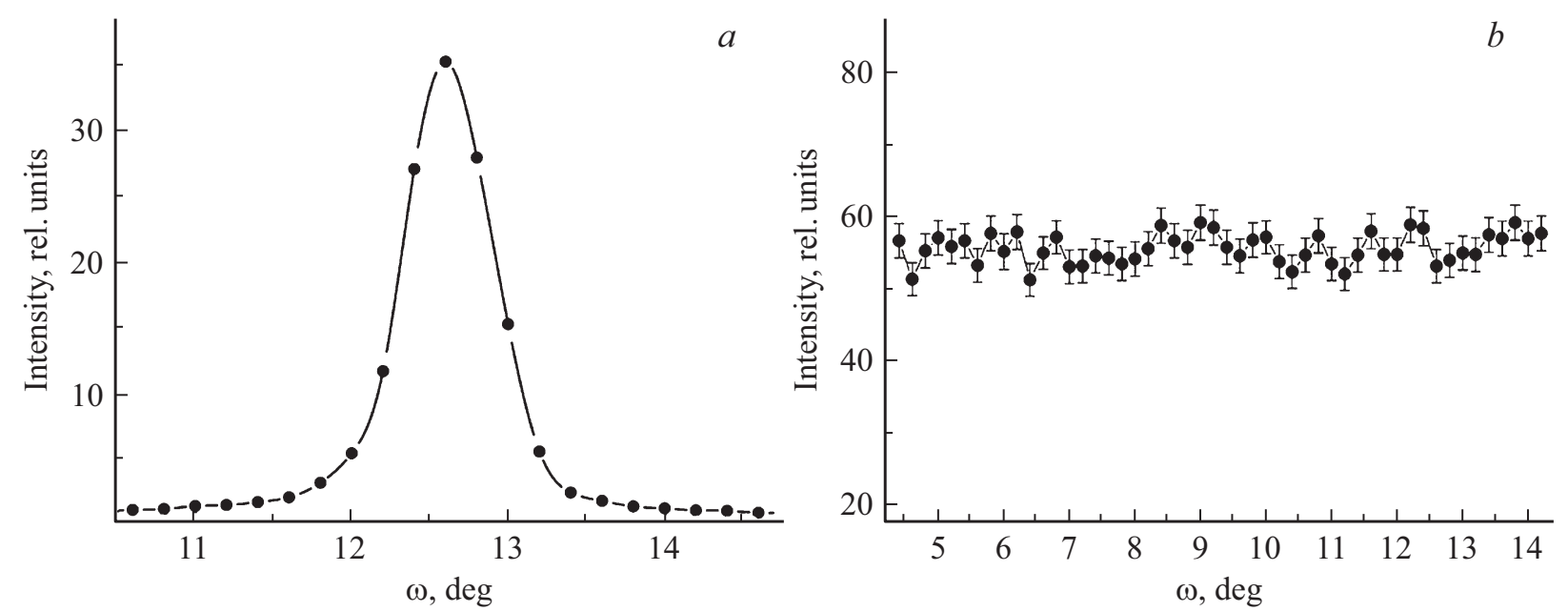

Рис. 6. $\omega$-сканы пика (111) при $2 \theta=25.2^{\circ}$ для отожженного образца (a), и фона рассеяния при $2 \theta=20.4^{\circ}$ для закаленного образца $(b)$.

рассеяния $(h k l)$ с нечетной суммой $h+k+l$ в дифрактограммах от сплава являются признаком ближнего упорядочения атомов примеси В2-типа. Ширина этих пиков на полувысоте указывает на то, что их размер не превышает длину двух ОЦК-ячеек сплава. Смещение их максимумов из расчетного положения в направлении больших углов рассеяния свидетельствуют о том, что ОЦК-ячейки, составляющие В2-кластер, существенно деформированы. Смещение сверхструктурных пиков от B2-фазы ранее наблюдались и в сплавах железа с кремнием [10,11] и алюминием [13], но тогда максимумы пиков смещались в сторону меньших углов. Было показано, что наблюдаемые особенности диффузного рассеяния объясняются присутствием в сплаве кластеров, состоящих преимущественно из двух ОЦК-ячеек, которые центрированы атомами кремния (или алюминия) и имеют общую грань, как показано на рис. 2.

Кратко резюмировать результаты, полученные из МНК-разложения дифрактограмм, можно следующим образом. В дифрактограммах закаленного образ-

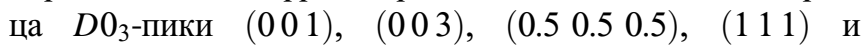
(1.5 1.51 .5$)$ гораздо слабее и шире, чем отожженного. Из рис. 4 и 5 видно, что они смещены из своих расчетных положений в дифрактограмме в направлении больших углов рассеяния. В обратной решетке величина смещения $\Delta l \approx 0.05$ вдоль оси [0 011$]$ и $\Delta h=\Delta k=\Delta l \approx 0.05$ вдоль оси [1 111$]$. Следовательно, параметр $D 0_{3}$-ячейки примерно на $5 \%$ меньше удвоенного параметра ОЦК-ячейки сплава и равен $\sim 0.55 \mathrm{~nm}$. Средний размер областей $D 0_{3}$-фазы в закаленном образце оценивается в $1.6 \mathrm{~nm}$, то есть равен длине трех ячеек $\mathrm{DO}_{3}$.

В дифрактограммах обоих образцов имеются широкие диффузные пики, расположенные вблизи позиций (001), (003) и (1 1 1). Они смещены из расчетных положений в направлении больших углов рассеяния в дифрактограммах вдоль оси $\left[\begin{array}{lll}0 & 0 & 1\end{array}\right]$ на $\Delta l \approx 0.25$ и на $\Delta h=\Delta k=\Delta l \approx 0.25$ вдоль оси [ [ 1111$]$ в индексах обратной решетки. Поскольку $\Delta h, \Delta k$ и $\Delta l$ примерно одинаковы, то можно предположить, что это сверхструктурные рефлексы от упорядоченных областей $B 2$-фазы, a точнее от B2-кластеров, локальное упорядочение в которых сопровождается деформациями решетки. Оцен-

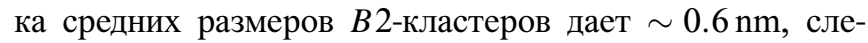
довательно, кластер состоит преимущественно из двух ячеек ОЦК-решетки. Это дает основание полагать, что B2-кластеры в $\mathrm{Fe}-\mathrm{Ga}-$ сплаве имеют одинаковую природу с наблюдавшимися ранее в богатых железом сплавах с кремнием или алюминием, приведенными на рис. $2[10,11,13]$.

Для определения строения В2-кластеров в сплаве железо-галлий проведены модельные расчеты с помощью пакета программ DISCUS [17]. Сначала моделировалась ОЦК-решетка кристалла, содержащая деформированные кластеры, то есть пары имеющих общую грань ОЦК-ячеек, центрированных атомами $\mathrm{Ga}$, аналогично

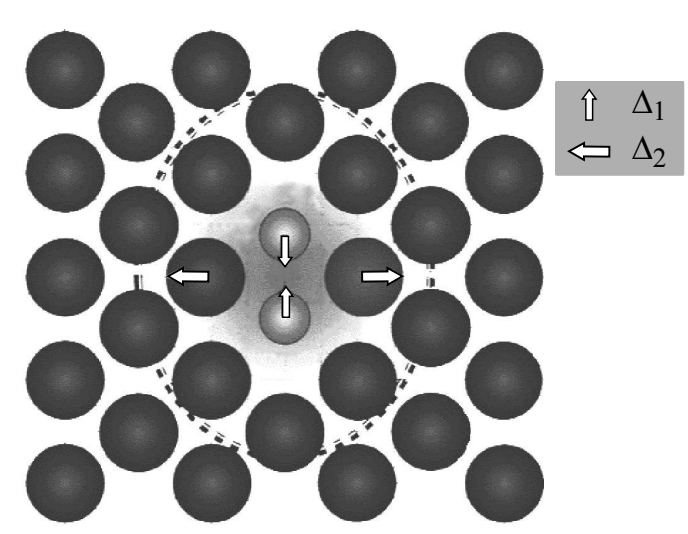

Рис. 7. Схема деформаций в ближайшем окружении пары атомов $\mathrm{Ga}$ в плоскости (110). Атомы галлия показаны меньшими окружностями, атомы железа - большими. Стрелками направления смещения атомов $\Delta_{1}$ и $\Delta_{2}$. 

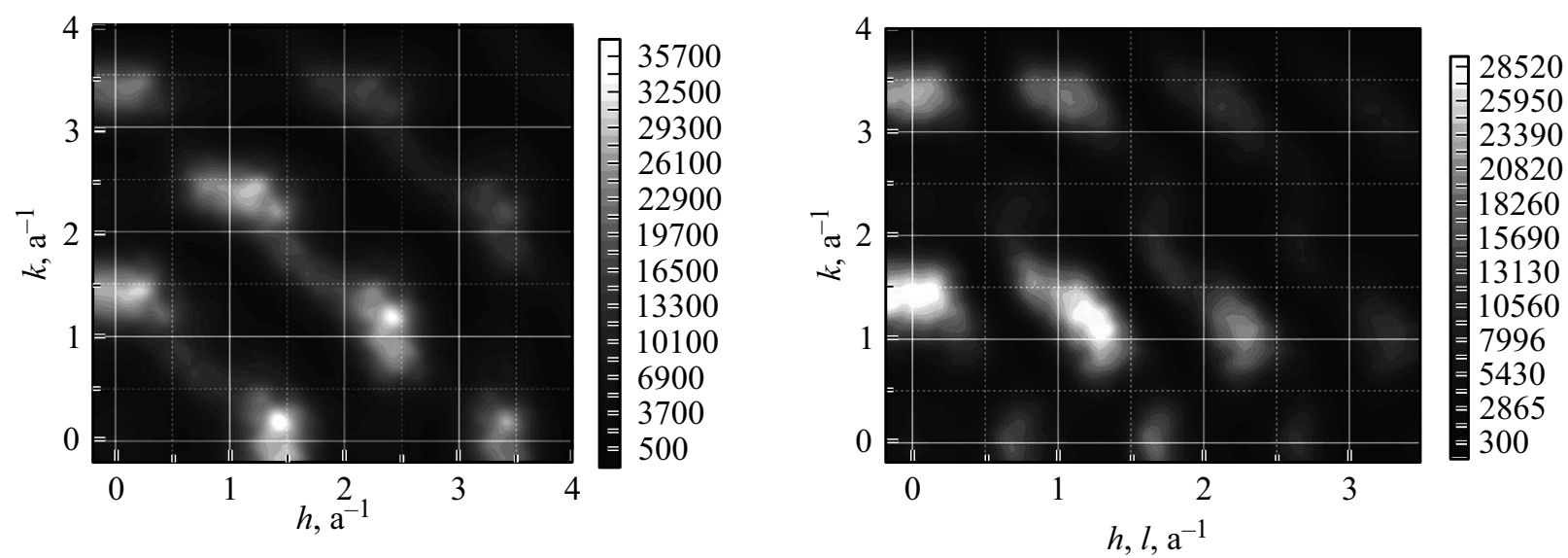

Рис. 8. Распределение интенсивности диффузного рассеяния в плоскости $(010)$ - слева и в плоскости $(1 \overline{1} 0)-$ справа. Расчет выполнен для модельной структуры со случайным распределением пар атомов галлия (B2-кластеров) с деформациями локального окружения, показанными на рис. 7.

тому, как это делалось в наших исследованиях железокремнистых [10,11] и железоалюминиевых [13] магнитомягких сплавов. Деформация кластера задавалась смещениями входящих в него атомов, симметричными относительно оси $\langle 100\rangle$, проходящей вертикально через центры атомов $\mathrm{Ga}$, как показано на рис. 7. Затем для модели „кристалла“ из большого числа атомов рассчитывалось распределение интенсивности диффузного рассеяния в плоскостях $(010)$ и (1 10$)$.

На первом этапе недеформированные В2-кластеры были случайным образом распределены в объеме, состоящем из $32 \times 32 \times 32=32768$ ОЦК-ячеек. При выполнении условия, что атом галлия не имеет атомов галлия в первой координационной сфере и может иметь только один атом галлия во второй сфере, тогда концентрация $\mathrm{Ga}$ атомов в модельном объеме достигает $\sim 6$ at.\%. Затем атомы галлия в кластере и ближайшие к ним атомы железа смещались из идеальных положений в решетке, как показано на рис. 7. Подбором величины смещений получено распределение интенсивности диффузного рассеяния в плоскостях (0 10) и (1 $\overline{1} 0)$ обратного пространства, наиболее соответствующее экспериментально наблюдаемым особенностям диффузного рассеяния от В2-кластеров или широким смещенным пикам (0 01 1), (0 03 ), выделенным на рис. 4, и (1 111$)$, выделенным на рис. 5 .

Картина рассеяния, приведенная на рис. 8, получена для относительных сдвигов атомов пары $\mathrm{Ga}-\mathrm{Ga}$ на $\Delta_{1}=0.01$ и относительных сдвигов атомов железа, находящихся в плоскости, перпендикулярной оси пары и проходящей через середину расстояния между ними, на $\Delta_{2}=0.02$. Направление сдвигов атомов $\Delta_{1}$ и $\Delta_{2}$ в B2-кластере показано стрелками на рис. 7. Сечение картины по оси $k$ на левом и правом рис. 8 соответствует экспериментальному скану вдоль оси [0 011$]$ и воспроизводит диффузные пики (001) и (003), сдвинутые в направлении больших углов рассеяния. Это сечение приведено на рис. 9, где для сравнения показан

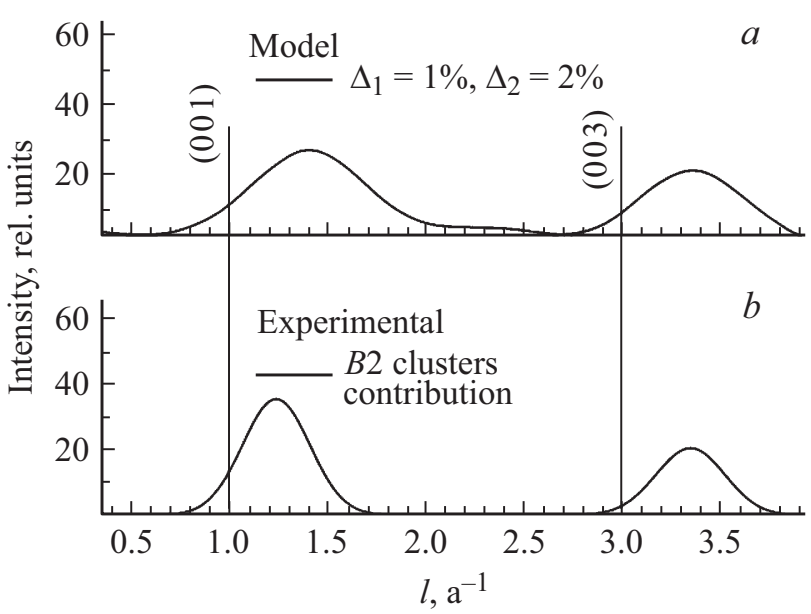

Рис. 9. Модельный расчет $(a)$ и вклад диффузного рассеяния от $B 2$-кластеров, полученный из разложения $\theta-2 \theta$-скана вдоль оси $\left[\begin{array}{lll}0 & 0 & 1\end{array}\right](b)$.

вклад от рассеяния на деформированных В2-кластерах, полученный из разложения $\theta-2 \theta$-скана вдоль оси [0 01 l].

Сечение картины по оси абсцисс на правом рис. 8 и по диагонали на левом соответствует экспериментальному скану по $\left[\begin{array}{lll}1 & 1 & 0\end{array}\right]$ и воспроизводит асимметрию фона пиков (110), (220) в экспериментальных дифрактограммах. Оно приведено на рис. 10 для двух вариантов сдвигов атомов $\Delta_{1}$ и $\Delta_{2}$, где вариант с $\Delta_{2}=2 \%$ более адекватен, чем с $\Delta_{2}=0$. Понятно, что модель локального упорядочения пар атомов $\mathrm{Ga}$, то есть модель $B 2$-кластеров, упрощена и не может хорошо описать все наблюдаемые в эксперименте особенности, да и структура сплава сложная, но даже такая модель отражает основные детали диффузного рассеяния, наблюдаемые в эксперименте.

Что касается FCC-фазы, то ее влияние на магнитные свойства сплава маловероятно, так как, судя по интенсивности соответствующих ей пиков в дифрактограммах, 


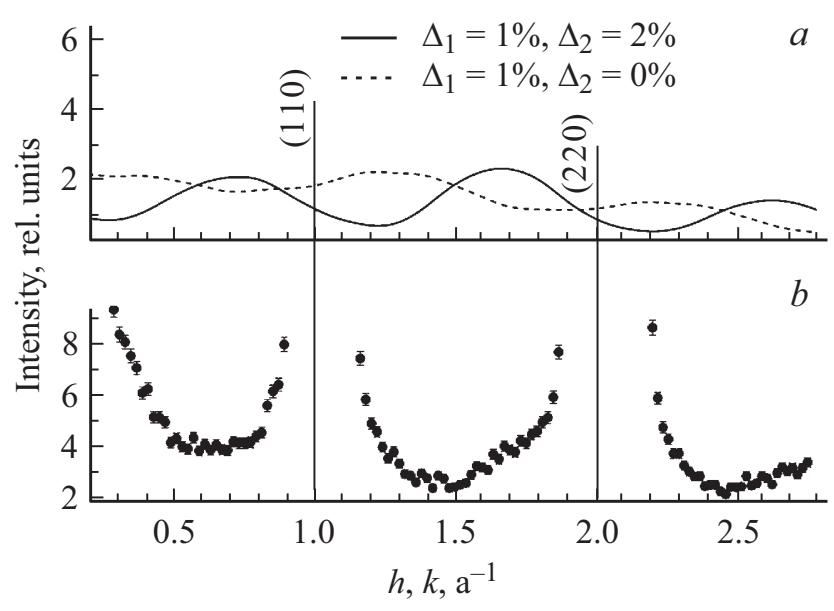

Рис. 10. Два варианта модельного расчета диффузного рассеяния вдоль оси $[110]$ с разными смещениями атомов в $B 2$-кластере $(a)$ и соответствующий скан образца сплава, подвергнутого закалке в воду $(b)$.

ее объемная доля в сплаве как после закалки, так и после отжига незначительна.

Таким образом, при отжиге в ферромагнитном состоянии упорядочение атомов в сплаве $\mathrm{Fe}-\mathrm{Ga}$ при 18 at.\% галлия идет в основном с образованием $D 0_{3}$-фазы и сохранением, или небольшим изменением, B2-кластеров. Если предположить, что после отжига в ферромагнитном состоянии весь объем образца поделён между двумя фазами: $D 0_{3}$-области и $B 2$-кластеры, то можно оценить относительные доли этих фаз в образце. Химический состав в областях $D 0_{3}$-фазы соответствует формуле $\mathrm{Fe}_{3} \mathrm{Ga}$ или 25 at.\% примесных атомов в ОЦК-железе. Средняя концентрация $\mathrm{Ga}$ в областях, занятых В2-кластерами, примерно 6 at.\%. Долями других фаз пренебрегаем. Тогда относительные доли $D 0_{3}$-фазы, $\delta_{D 03}$, и $B 2$-фазы, $\delta_{B 2}$, в сплаве связаны уравнениями

$$
\begin{gathered}
\delta_{D 03} \cdot C_{D 03}+\delta_{B 2} \cdot C_{B 2}=C_{\mathrm{Ga}}, \\
\delta_{D 03}+\delta_{B 2}=1,
\end{gathered}
$$

где $C_{D 03}=0.25$ и $C_{B 2}=0.06-$ концентрации галлия в областях $D_{0}$-фазы и в $B 2$-кластерах, соответственно, а $C_{\mathrm{Ga}}-$ средняя концентрация $\mathrm{Ga}$ в сплаве. Тогда

$$
\delta_{B 2}=\left(C_{D 03}-C_{\mathrm{Ga}}\right) /\left(C_{D 03}-C_{B 2}\right) .
$$

Подстановка концентраций дает $\delta_{D 03} \approx 0.63$ и максимальную оценку $\delta_{B 2} \approx 0.37$, то есть около $40 \%$ объема сплава могут занимать области с B2-кластерами, и около $60 \%$ - области $D 0_{3}$-фазы. Если при средней концентрации галлия 18 at.\% все атомы Ga входят в одну $D 0_{3}$-фазу, то ее максимальная доля $\sim 70 \%$ от объема образца. Понятно, что в реальных сплавах не все атомы $\mathrm{Ga}$ входят в $D 0_{3}$-фазу и $B 2$-кластеры. Могут существовать области A2-фазы и даже чистого железа, но в дифрактограммах вклад от них складывается с сильными пиками от ОЦК-решетки сплава.

\section{4. Заключение}

Проведенные рентгендифракционные исследования атомной структуры монокристаллов сплава железогаллий с содержанием галлия около 18 at.\% показали, что в образцах сплава имеет место упорядочение атомов галлия. Тип атомного упорядочения в областях кристалла и размер областей зависят от условий термической обработки сплава. В дифрактограммах образца, закаленного в воду после выдержки в парамагнитном состоянии, кроме сильных пиков от основной ОЦК-структуры наблюдается вклад диффузного рассеяния от фаз $B 2$, $\mathrm{DO}_{3}$ и FCC. Отжиг в ферромагнитном состоянии не приводит к заметному изменению вклада в рассеяние от небольших (субнаноразмерных, $\sim 0.6 \mathrm{~nm}$ ) кластеров $B 2$-фазы, в то время как $D 0_{3}$ - и FCC-пики становятся узкими, их интенсивность увеличивается, причем у $D 0_{3}$-пиков почти на два порядка. FCC-фаза определена как кубическая гранецентрированная. Оси FCC-ячейки совпадают с осями ОЦК-решетки сплава. Поскольку ее объемная доля незначительна, то и ее влияние на магнитные свойства маловероятно.

В работе [5] предполагается, что как раз особенности структуры сплавов железо-галлий и определяют магнитоупругие свойства, то есть магнитострикция уменьшается с ростом объемной доли $D 0_{3}$-фазы. Именно это и наблюдается. В закаленном образце объемная доля $D 0_{3}$-фазы (области размером $1.6 \mathrm{~nm}$ или длиной в три элементарные $D 0_{3}$-ячейки) мала, но после отжига в ферромагнитном состоянии формируется дальний $D 0_{3}$-порядок. После закалки коэффициент тетрагональной магнитострикции $3 / 2 \lambda_{100}$ принимает максимальное значение около $400 \times 10^{-6}$ (рис. 1). После отжига в ферромагнитном состоянии области $D 0_{3}$-фазы значительно увеличиваются в объеме и степени порядка. В своем максимальном значении объемная доля областей $D 0_{3}$-фазы при концентрации $\mathrm{Ga} 18 \%$ может достигать $\sim 70 \%$. Если учесть присутствие B2-кластеров, то относительные объемные доли могут составить около 60 и $40 \%$ для областей $D 0_{3}$-фазы и $B 2$-кластеров, соответственно. Если предположить, что $D 0_{3}$-фаза оказывает негативное влияние на магнитоупругие свойства материала, то становится понятной разница, достигающая 25\%, величины коэффициента тетрагональной магнитострикции у закаленного и медленно охлажденного образцов сплава при 19 at.\% Ga.

Кластеры B2-фазы (пары примесных атомов - вторые соседи в ОЦК-решетке железа), вероятно, существуют в сплавах при меньших концентрациях галлия, независимо от условий термообработки. Поскольку вероятность образовать такую пару пропорциональна квадрату концентрации атомов галлия, $C_{\mathrm{Ga}}$, то при наличии тенденции к их образованию количество пар $\mathrm{Ga}-\mathrm{Ga}$ должно быть пропорционально $C_{\mathrm{Ga}}^{2}$. Поэтому, возможно, присутствие $B 2$-кластеров, их положительное влияние на увеличение магнитострикции, определяют 
квадратичный рост коэффициента $\lambda_{100}$ с ростом $C_{\mathrm{Ga}}$ от 0 до 17 at.\%.

Даже для сильно упрощенной модели B2-кластера анизотропной формы, в котором атомы галлия и железа сдвинуты из узлов идеальной решетки, как показано на рис. 7, наши расчеты воспроизводят основные особенности, наблюдаемые в дифрактограммах. В частности, значительное смещение из расчетных положений в сторону больших углов рассеяния сверхструктурных $B 2$-пиков с нечетной суммой индексов $h, k$ и $l$ из-за локальных деформаций решетки, а также асимметрию фона вблизи ОЦК-пиков ( 110$)$ и (2 20 ).

Результаты исследований согласуются с результатами первопринципных расчетов [4], где рассматриваются процессы формирования ближнего порядка в $\mathrm{Fe}-\mathrm{Ga}-\mathrm{sвердых} \mathrm{растворах.} \mathrm{Показано,} \mathrm{что} \mathrm{межатомное}$ взаимодействие зависит от магнитного состояния матрицы. При переходе в ферромагнитное состояние эффективная энергия взаимодействия пары $\mathrm{Ga}-\mathrm{Ga}$ по сравнению с парамагнитным состоянием уменьшается примерно в два раза в случае первых соседей, увеличивается для вторых и достигает минимума для третьих. Поэтому атомный ближний порядок $B 2$ формируется предпочтительно в парамагнитном состоянии $\left(T>T_{\mathrm{C}}\right)$, a атомный ближний порядок $\mathrm{DO}_{3}$ соответствует равновесному состоянию сплава в ферромагнитном состоянии $\left(T<T_{\mathrm{C}}\right)$.

Можно предположить, что в сплавах системы $\mathrm{Fe}-\mathrm{Ga}$ действует механизм, аналогичный предложенному в [18], где рассматривается изменение упорядочения атомов в богатых железом сплавах $\mathrm{Fe}-\mathrm{Si}$. B2-пары вторых соседей атомов $\mathrm{Si}$ (рис. 2), образующиеся в парамагнитном состоянии, сохраняются при охлаждении благодаря образованию ими устойчивого комплекса с вакансией, препятствующего диссоциации пары диффузионным путем. Тогда и в сплавах $\mathrm{Fe}-\mathrm{Ga}$ для возникновения локального порядка типа $D 0_{3}$ при отжиге в ферромагнитном состоянии необходима дополнительная активация диффузионного процесса, т.е. преодоления энергетического барьера $0.3-0.5 \mathrm{eV}$. Поэтому перестройка ближнего порядка $B 2$ в $D 0_{3}$ не происходит спонтанно при понижении температуры ниже точки Кюри, а развивается постепенно в процессе длительного отжига при $T<T_{\mathrm{C}}$. Скорее всего, объемная доля $D 0_{3}$-фазы при высоких концентрациях галлия от 17 до 20 at.\% увеличивается за счет диффузии в нее атомов галлия из неупорядоченной А2-фазы.

\section{Список литературы}

[1] J.R. Cullen, A.E. Clark, M. Wun-Fogle, J.B. Restor, T.A. Lograsso. J. Magn. Magn. Mater. 226-230, 948 (2001). https://doi.org/10.1016/S0304-8853(00)00612-0

[2] A.E. Clark, K.B. Hathaway, M. Wun-Fogle, J.B. Restorff, T.A. Lograsso, V.M. Keppens, G. Petculescu, R.A. Taylor. J. Appl. Phys. 938621 (2003).

https://doi.org/10.1063/1.1540130
[3] O. Ikeda, R. Kainuma, I. Ohnuma, K. Fukamichi, K. Ishida. J. Alloys Compd. 347, 198 (2002). https://doi.org/10.1016/S0925-8388(02)00791-0

[4] М.В. Петрик, О.И. Горбатов, Ю.Н. Горностырев. Письма в ЖЭТФ 98 (12), 912 (2013). DOI: https://doi.org/10.7868/S0370274X13240107

[5] R. Wu. J. Appl. Phys. 91, 7358 (2002). https://doi.org/10.1063/1.1450791

[6] J. Cullen, P. Zhao, M. Wuttig. J. Appl. Phys. 101, 123922 (2007). https://doi.org/10.1063/1.2749471

[7] J. Boisse, H. Zapolsky, A.G. Khachaturyan. Acta Mater. 59, 2656 (2011). https://doi.org/10.1016/j.actamat.2011.01.002

[8] T.A. Lograsso, E.M. Summers. Mater. Sci. Eng. A 416, 240 (2006). https://doi.org/10.1016/j.msea.2005.10.035

[9] M. Huang, T.A. Lograsso. Appl. Phys. Lett. 95, 171907-1 (2009). https://doi.org/10.1063/1.3254249

[10] Yu.P. Chernenkov, N.V. Ershov, V.A. Lukshina, V.I. Fedorov, B.K. Sokolov. Physica B: Condens. Matter. 396, 1-2, 220 (2007). Doi: 10.1016/j.physb.2007.04.008

[11] Н.В. Ершов, Ю.П. Черненков, В.А. Лукшина, В.И. Федоров. ФТТ 51, 3, 417 (2009). DOI: $10.1134 / \mathrm{S} 1063783409030019$

[12] Н.В. Ершов, Ю.П. Черненков, В.А. Лукшина, В.И. Федоров. ФТТ 54, 9, 1813 (2012). DOI: $101134 / \mathrm{S} 1063783412090107$

[13] Н.В. Ершов, Ю.П. Черненков, В.А. Лукшина, О.П. Смирнов. ФТТ 60, 9, 1619 (2018).

[14] Ю.П. Черненков, В.И. Федоров, В.А. Лукшина, Б.К. Соколов, Н.В. Ершов. ФММ 100, 3, 39 (2005). http://impo.imp.uran.ru/fmm/Electron/vol100_3/abstract6.html

[15] C.J. Quinn, P.J. Grundy, N.J. Mellors. J. Magn. Magn. Mater. 361, 74 (2014). https://doi.org/10.1016/j.jmmm.2014.02.004

[16] B.D. Cullity, S.R. Stock. Elements of X-Ray Diffraction. Prentice-Hall Inc., N. Y. (2001). 531 p. https://www.scholars.northwestern.edu/en/publications/ elements-of-x-ray-diffraction-third-edition

[17] Th. Proffen, R.B. Neder. J. Appl. Crystallogr. 30, 171 (1997). Doi: $10.1107 / \mathrm{S} 002188989600934 \mathrm{X}$

[18] О.И. Горбатов, А.Р. Кузнецов, Ю.Н. Горностырев, А.В. Рубан, Н.В. Ершов, В.А. Лукшина, Ю.П. Черненков, В.И. Федоров. ЖЭТФ 139, 5, 969 (2011). DOI: $10.1134 / \mathrm{S} 1063776111040066$

Редактор Т.Н. Василевская 